\title{
Manganese modified structural and optical properties of zinc soda lime silica glasses
}

\begin{abstract}
A series of MnO-doped zinc soda lime silica glass systems was prepared by a conventional melt and quenching technique. In this study, the x-ray diffraction analysis was applied to confirm the amorphous nature of the glasses. Fourier transform infrared spectroscopy shows the glass network consists of $\mathrm{MnO} 4, \mathrm{SiO} 4$, and $\mathrm{ZnO} 4$ units as basic structural units. The glass samples under field emission scanning electron microscopy observation demonstrated irregularity in shape and size with glassy phase-like structure. The optical absorption studies revealed that the optical bandgap (Eopt) values decrease with an increase of $\mathrm{MnO}$ content. Through the results of various measurements, the doping of $\mathrm{MnO}$ in the glass matrix had effects on the performance of the glasses and significantly improved the properties of the glass sample as a potential host for phosphor material.
\end{abstract}

Keyword: Manganese modified structural; Optical properties; Zinc soda lime silica glasses 